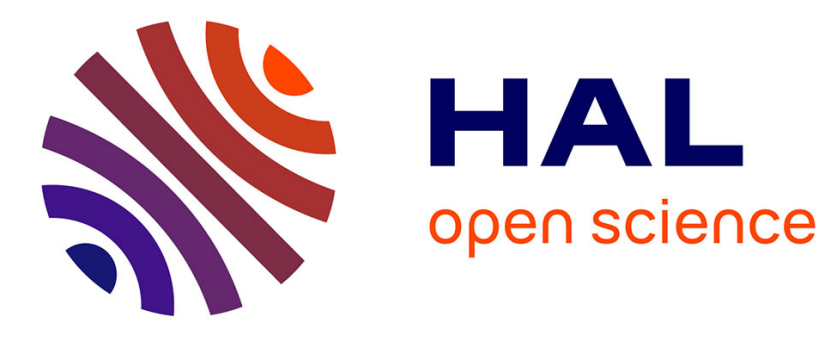

\title{
Hybrid Check Node Architectures for NB-LDPC Decoders
}

Cédric Marchand, Emmanuel Boutillon, Hassan Harb, Laura Conde-Canencia, Ali Al Ghouwayel

\section{- To cite this version:}

Cédric Marchand, Emmanuel Boutillon, Hassan Harb, Laura Conde-Canencia, Ali Al Ghouwayel. Hybrid Check Node Architectures for NB-LDPC Decoders. IEEE Transactions on Circuits and Systems I: Regular Papers, 2019, 66 (2), pp.869-880. 10.1109/TCSI.2018.2866882 hal-01874967

\section{HAL Id: hal-01874967 https://hal.science/hal-01874967}

Submitted on 15 Sep 2018

HAL is a multi-disciplinary open access archive for the deposit and dissemination of scientific research documents, whether they are published or not. The documents may come from teaching and research institutions in France or abroad, or from public or private research centers.
L'archive ouverte pluridisciplinaire HAL, est destinée au dépôt et à la diffusion de documents scientifiques de niveau recherche, publiés ou non, émanant des établissements d'enseignement et de recherche français ou étrangers, des laboratoires publics ou privés. 


\title{
Hybrid Check Node Architectures for NB-LDPC Decoders
}

\author{
Cédric Marchand, Member, IEEE, Emmanuel Boutillon, Senior Member, IEEE, Hassan Harb, \\ Laura Conde-Canencia, Member, IEEE, and Ali Al Ghouwayel, Member, IEEE,
}

\begin{abstract}
This paper proposes a unified framework to describe check node architectures of Non-Binary LDPC decoders. Forward-Backward, Syndrome-Based and Pre-sorting approaches are first described. Then, they are hybridized in an effective way to reduce the amount of computation required to perform a check node. This work is specially impacting check nodes of high degrees (or high coding rates). Results of $28 \mathrm{~nm}$ ASIC post-synthesis for a check node of degree 12 (i.e. code rate of 5/6 with a degree of variable equal to 2) are provided for NBLDPC over GF(64) and GF(256). While simulations show almost no performance loss, the new proposed Hybrid implementation check node increases the hardware and the power efficiency by a factor of six compared to the classical Forward-Backward architecture. This leads to the first ever reported implementation of a degree 12 check node over $G F(256)$ and these preliminary results open the road to high decoding throughput, high rate, and high order Galois Field NB-LDPC decoder with reasonable hardware complexity.
\end{abstract}

Index Terms-NB-LDPC, Check Node, syndrome-based, VLSI, Foward-Backward.

\section{INTRODUCTION}

$\mathbf{L}$ OW-Density Parity-Check (LDPC) codes [1] have now been adopted for a wide range of standards (WiMAX, WiFi, DVB-C, DVB-S2X, DVB-T2) because of their nearchannel-capacity performance. However, the capacity achieving performance is obtained for long codeword lengths and LDPC codes start to show their weakness when considering short and moderate codeword lengths. In the last decade significant research effort has been devoted to the extension of LDPC codes to high-order Galois $\operatorname{GF}(q)(q>2$ is the order of the GF). This family of codes, named Non-Binary (NB) LDPC codes, show strong potential for error correction capability with moderate and short codeword lengths [2]. This is mainly due to the fact that NB-LDPC codes present higher girths than their binary counterparts, and thus present better decoding performance with message passing algorithms. Also, the NB nature of such NB-LDPC codes makes them suitable for high-spectral-efficiency modulation schemes where the constellation symbols are directly mapped to $\mathrm{GF}(q)$ symbols [3]. This mapping bypasses the marginalization process of binary LDPC codes that causes information loss. NB-LDPC codes becomes serious competitors of classical binary LDPC and Turbo Codes in future wireless communication and digital video broadcasting standards.

The main drawback of NB-LDPC codes is related to their high decoding complexity. In the NB-LDPC decoder each message exchanged between the processing nodes is an array of values, each one corresponding to a GF element. From an implementation point of view, this leads to a highly increased complexity compared to binary LDPC decoding.

A straightforward implementation of the Belief-Propagation (BP) algorithm for NB-LDPC results in a computational complexity of the order of $O\left(q^{2}\right)$ [2]. The Extended MinSum (EMS) algorithm, which is an extension of the wellknown Min-Sum algorithm from the binary to the NB domain, represents an interesting compromise between hardware complexity and error correction performance [4] [5]. As the Check Node $(\mathrm{CN})$ processing constitutes the computational bottleneck in the EMS decoding, much research work has focused on its complexity reduction. Currently, state-of-theart architectures apply the Forward-Backward (FB) algorithm [5] to process the CN. With this approach, a serial calculation is carried out to reduce the hardware cost and to reuse results from intermediate Elementary CN (ECN). However, the FB $\mathrm{CN}$ structure suffers from high latency and low throughput. The Trellis-EMS (T-EMS) introduced in [6] avoids the long latency of the FB computation but its hardware complexity highly increases with $q$ when a parallel implementation is considered. The complexity of the T-EMS was reduced with the one-minimum T-EMS [7] and the Trellis Min-Max (T-MM) [8] [9] algorithms.

The SB algorithm, recently presented in [10] [11], is an efficient method to perform a parallel computation of the $\mathrm{CN}$ when $q \geq 16$. This architecture was used for the first reported implementation of a $\mathrm{GF}(256) \mathrm{CN}$ processor with degree $d_{c}=4$ [12]. However, the complexity of the SB CN algorithm is dominated by the number of syndromes to be computed, which increases quadratically with $d_{c}$, limiting its interest for high coding rates, i.e. for high $d_{c}$ values. Recently, we showed that sorting the input vector of the $\mathrm{CN}$ according to a reliability criteria [13] [14] allows significant reduction of the hardware complexity of the $\mathrm{CN}$ architecture without affecting performance. This technique, called "presorting", has been successfully combined with the SB CN, leading to the PreSorted-SB CN (or PS-SB CN) algorithm [13] and with the FB CN, leading to the PS-FB CN [14].

The goals of this paper are twofold. The first one is to synthesize several contributions published in conference papers in an unified framework. The second goal is to present a simplification of the Extented Forward (EF) architecture and several original hybridizations of existing architectures to further reduce the hardware complexity of the $\mathrm{CN}$ and to derive efficient hardware implementations for high-order GF and/or high $\mathrm{CN}$ degrees. The pre-sorting is the key to understand the efficiency of the Hybrid architecture compared 
TABLE I

CN ARCHITECTURES BASED ON THE EMS ALGORITHM.

\begin{tabular}{c|c||c|c}
\hline \multicolumn{2}{c||}{ CN architectures } & \multicolumn{2}{c}{ Input processing } \\
\hline & Full form & Normal & PreSorted (PS) \\
\hline \hline T-EMS & Trellis EMS & {$[6][7]$} & - \\
\hline T-MM & Trellis Min-Max & {$[8][9]$} & - \\
\hline FB & Forward-Backward & {$[5][15][16]$} & {$[14]^{*}$} \\
\hline SB & Syndrome-Based & {$[10]^{*}[11]^{*}[12]$} & {$[13]^{*}$} \\
\hline EF & Extended Forward-SB & {$[17]^{*}$} & {$[17]^{*}$, this paper } \\
\hline HB & Hybrid & - & This paper \\
\hline
\end{tabular}

to previous algorithm. Pre-sorting allows to match locally the processing algorithm (SB, EF or FB) with the dynamic of incoming and intermediate messages. Table I presents the names of the different existing and proposed architectures. In this Table, the references with an asterisk correspond to previous contributions of the authors.

The paper is structured as follows: Section II recalls the structure of a NB-LDPC code and presents the EMS decoding algorithm. Section III gives a survey of the existing practical EMS architectures. Section IV presents several original hybrid architectures and Section $\mathrm{V}$ provides synthesis and performance results. Finally, conclusions are drawn in Section VI.

\section{NB-LDPC NOTATIONS AND DECODING}

This section first recalls the principles and notations of NBLDPC codes. Then, the EMS algorithm is described.

\section{A. NB-LDPC code}

A NB-LDPC code is a linear block code defined by a sparse parity-check matrix $\mathbf{H}$ of size $M \times N$. The $M$ rows of the matrix $\mathbf{H}$ refer to $M$ parity check equations. The $i^{t h}$ parity check equation is defined as $d_{c}(i)$ non-zero $\operatorname{GF}(q)$ values $\left\{h_{i, j_{i}(k)}\right\}_{k=1 \ldots d_{c}(i)}$ of the $i^{t h}$ line of the parity check matrix as

$$
\sum_{k=1}^{d_{c}(i)} h_{i, j_{i}(k)} x\left(j_{i}(k)\right)=0,
$$

where $\left\{x\left(j_{i}(k)\right)\right\}_{k=1 \ldots d_{c}(i)}$ is a subset of size $d_{c}(i)$ of the $N$ $\mathrm{GF}(q)$ symbols of the code. A NB-LDPC code is regular if the number $d_{c}$ of non-zero values per row and the number $d_{v}$ of non-zero values per column are constants. Efficient NB-LDPC coding schemes can be constructed with $d_{v}=2$ [18]. In this case, assuming a full-rank parity-check matrix $\mathbf{H}$, the rate of the code is $r=1-2 / d_{c}$.

\section{B. EMS algorithm}

For simplicity, the EMS algorithm is described only at the CN level. The reader can refer to [19] for a complete description of the whole decoding process including the variable and edge nodes.

Fig. 1 shows a $\mathrm{CN}$ and its $d_{c}=4$ conected Varible Nodes (VN). Let us define a $\mathrm{CN}$ equation of degree $d_{c}$ in $\mathrm{GF}(q)$ as $e_{1} \oplus e_{2} \oplus e_{3} \oplus \ldots \oplus e_{d_{c}}=0$, where $\oplus$ represents the addition over $\operatorname{GF}(q)^{1}$ and $e_{k}=h_{i, k} x_{k}$. Each input $e_{i}$ is a variable

\footnotetext{
${ }^{1}$ or, equivalently, subtraction in $\operatorname{GF}(q)$.
}

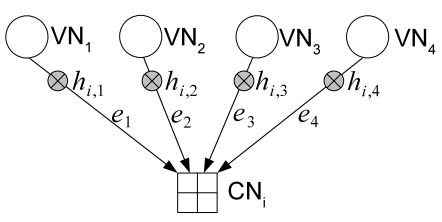

Fig. 1. Message notation on a $\mathrm{CN}$

defined in $\mathrm{GF}(q)$ and its associated a priori information is the discrete probability distribution $P\left(e_{i}=x\right), x \in \mathrm{GF}(q)$. Each element of the probability distribution $E$ associated to $e$ can be expressed in the logarithmic domain as the Log-Likelihood Ratio (LLR) $e^{+}(x)$ defined as

$$
e^{+}(x)=-\log \left(\frac{P(e=x)}{P(e=\bar{x})}\right),
$$

where $\bar{x}$ is the hard decision on $e$ obtained with the maximum likelihood criterion, i.e. $\bar{x}=\arg \max _{x \in \mathrm{GF}(q)} P(e=x)$. From this LLR definition: $e^{+}(\bar{x})=0$ and $\forall x \in \mathrm{GF}(q), e^{+}(x) \geq 0$. The distribution (or message) $E$ associated to $e$ is thus $E=$ $\left\{e^{+}(x)\right\}_{x \in \mathrm{GF}(q)}$.

The EMS algorithm is an extention of the Min-Sum aiming at reducing the complexity of NB-LDPC decoders. Its main characteristic is the truncation of the messages $E$ from $q$ to the $n_{m}$ most reliable values $\left(n_{m} \ll q\right)$. At the $\mathrm{CN}$ level, each incoming message $U$ is composed of $n_{m}$ couples sorted in increasing order of LLRs (note that a high LLR value means low reliability). In Fig. 1, each input $U$ of the $\mathrm{CN}$ is a list $\{U[j]\}_{j=0 \ldots n_{m}-1}$ of couples $U[j]=\left(U^{+}[j], U^{\oplus}[j]\right)$, where $U^{+}[j]$ is the $(j+1)^{t h}$ smallest LLR value of $E$ and $U^{\oplus}[j]$ is its associated GF element, i.e., $e^{+}\left(U^{\oplus}[j]\right)=U^{+}[j]$. Note also that $U^{+}[0]=0, U^{\oplus}[0]=\bar{x}$, and that $j \leq j^{\prime} \Rightarrow U^{+}[j] \leq$ $U^{+}\left[j^{\prime}\right]$. The same representation is used for each output $V$ of the $\mathrm{CN}$.

The EMS algorithm can be described in two steps:

\section{Computation:}

$$
v_{i}^{+}(x)=\min \left\{\sum_{i^{\prime}=1, i^{\prime} \neq i}^{d_{c}} U_{i^{\prime}}^{+}\left[j_{i^{\prime}}\right] \mid \bigoplus_{i^{\prime}=1, i^{\prime} \neq i}^{d_{c}} U_{i^{\prime}}^{\oplus}\left[j_{i^{\prime}}\right]=x\right\},
$$

where $j_{i^{\prime}} \in\left\{0,1, \ldots n_{m}-1\right\}$ for $i^{\prime}=1,2, \ldots d_{c}, i^{\prime} \neq i$.

Sorting: the $v_{i}^{+}(x)$ are sorted in increasing order and the first $n_{m}$ smallest values are kept to generate the output vector $V_{i}$.

Note that at least one term in (3) is the summation of zero values since $u_{i}^{+}[0]=0$. Moreover, some values of $x$ may not be available due to the reduced search space.

As the direct computation of (3) implies a prohibitive number of calculations, different approaches have been proposed for the EMS CN processing. They are presented in the following Section.

\section{PRACTICAL IMPLEMENTATIONS OF EMS CN PROCESSING}

This section review the two state-of-the-art implementations of the EMS algorithm: the Forward-Backward (FB) and the Syndrome-Based (SB). Then, the presorting technique 


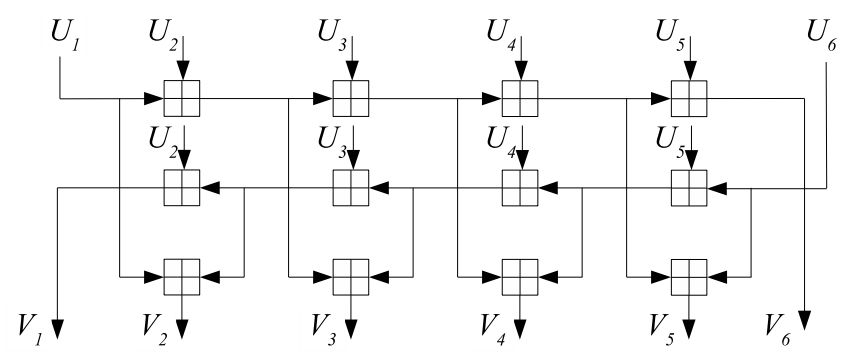

Fig. 2. FB CN processing with $d_{c}=6$

is introduced to show that sorting the $\mathrm{CN}$ input messages can lead to significant saving in terms of computations and implementation hardware.

\section{A. Forward-Backward CN processing}

The FB CN algorithm exploits the commutative and associative properties of the addition in $\operatorname{GF}(q)$ and factorizes (3) using a set of 2-input 1-output ECN. The $\mathrm{CN}$ processing is splitted into three layers: forward, backward and merge; each one containing $d_{c}-2$ ECNs [5]. An ECN processes a single output $C$ as a function of two inputs $A$ and $B$. Fig. 2 shows the resulting structure for a $\mathrm{FB} \mathrm{CN}$ with $d_{c}=6$ inputs using $\left(d_{c}-2\right) \times 3=12$ ECNs; each ECN being represented by a block $\boxplus$. Intermediate results of the ECNs are reused in the later stages, avoiding re-computations and thus reducing the amount of processing. Several reported hardware implementations of NB-LDPC decoders use this efficient FB architecture [20] [21].

The ECN processing [20] can be described in three steps.

1) Addition: for each couple of index $(a, b) \in$ $\left\{0,1, \ldots, n_{m}-1\right\}^{2}$, the output tuple

$$
C_{a, b}=\left(c^{+}(x), x\right)=\left(A^{+}[a]+B^{+}[b], A^{\oplus}[a] \oplus B^{\oplus}[b]\right)
$$

is computed.

2) Sorting: the couples $\left(c^{+}(x), x\right)$ are sorted in increasing order of $c^{+}(x)$. The output vector $C$ contains the first $n_{m}$ ordered couples corresponding to the first $n_{m}$ smallest values of $c^{+}(x)$.

3) Redundancy elimination: if two couples $(a, b)$ and $\left(a^{\prime}, b^{\prime}\right)$ correspond to the same GF value, i.e., $A^{\oplus}[a] \oplus$ $B^{\oplus}[b]=A^{\oplus}\left[a^{\prime}\right] \oplus B^{\oplus}\left[b^{\prime}\right]$, the one with higher LLR is suppressed during this Redundancy Elimination (RE) step. In order to generate at least $n_{m}$ valid outputs with a high probability, a number $n_{o p}>n_{m}$ outputs is generated by each ECN (typically, $n_{o p}=n_{m}+2$ ) [4].

Note that since $A^{+}[0]=0$ and $B^{+}[0]=0$, the first output value of $C$ is $C(0)=\left(0, A^{\oplus}[0]+B^{\oplus}[0]\right)$. For the sake of clarity and for the rest of the paper, the three ECN steps are represented by $\boxplus$ as:

$$
C=A \boxplus B .
$$

In [20], it is shown that (4) needs to be evaluated only for indexes $(a, b)$ that verify $(a+1)(b+1) \leq n_{o p}$. In fact, since

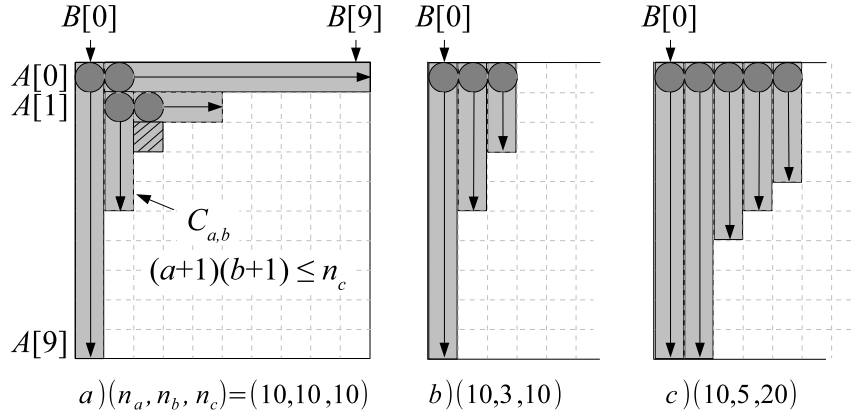

Fig. 3. S-bubble ECN and generalized S-bubble ECN

vectors $A^{+}$and $B^{+}$are sorted in increasing order of LLR, any couple $\left(a^{\prime}, b^{\prime}\right)$ verifying $\left(0 \leq a^{\prime} \leq a\right)$ and $\left(0 \leq b^{\prime} \leq b\right)$ gives $C_{a^{\prime}, b^{\prime}}^{+} \leq C_{a, b}^{+}$. Consequently, there are at least $(a+1)(b+1)$ couples $\left(a^{\prime}, b^{\prime}\right)$ verifying $C_{a^{\prime}, b^{\prime}}^{+} \leq C_{a, b}^{+}$. In other words, if $(a+1)(b+1)>n_{o p}$, then $C_{a, b}$ does not belong to the set of the $n_{o p}$ smallest values and thus, does not need to be evaluated.

As proposed in [14], the notion of potential bubbles is proposed by specifying the index variation ranges $n_{a}$ and $n_{b}$ of the two entries $A$ and $B$, i.e. $0 \leq a<n_{a}$ and $0 \leq b<n_{b}$ and the one of the output $C$ as $n_{c}$, i.e. $0 \leq c<n_{c}$. Note that $n_{a}$ and $n_{b}$ should be smaller than or equal to $n_{c}$.In Fig. 3(a), the subset of potential bubbles is represented in grey for $n_{m}=n_{o p}=10$. Fig. 3(b) and Fig. 3(c) show the potential bubbles when $\left(n_{a}, n_{b}, n_{c}\right)=(10,3,10)$ and $\left(n_{a}, n_{b}, n_{c}\right)=(10,5,20)$, respectively.

A serial hardware implementation of the Bubble $\mathrm{CN}$ architecture was presented in [20]. Suboptimal versions considering only the subset of the most probable potential bubbles (the first two rows and two columns) were presented in [20], [21] and [22].

\section{B. Syndrome-based CN processing}

The SB CN algorithm relies on the definition of a deviation path and its associated syndrome. In the sequel, $n_{m, i n}$ (resp. $n_{m, \text { out }}$ ) refers to the size of the input (resp. output) vector of a $\mathrm{CN}$.

A deviation path, denoted by $\delta$, is defined as a $d_{c}$-tuple of integer values, i.e. $\delta=\left(\delta(1), \delta(2), \ldots, \delta\left(d_{c}\right)\right)$, with $\delta(i) \in$ $\left\{0,1, \ldots, n_{m, i n}-1\right\}, i=1,2 \ldots, d_{c}$. A syndrome associated to a deviation path $\delta$ is denoted by $S(\delta)$ and defined as the 3-tuple $\left(S^{+}(\delta), S^{\oplus}(\delta), S^{D}[\delta]\right)$ with

$$
\begin{gathered}
S^{+}(\delta)=\sum_{i=1}^{d_{c}} U_{i}^{+}[\delta(i)], \\
S^{\oplus}(\delta)=\bigoplus_{i=1}^{d_{c}} U_{i}^{\oplus}[\delta(i)], \\
S^{D}[\delta][i]=\left\{\begin{array}{ll}
0, & \text { if } \delta(i)=0 \\
1, & \text { otherwise }
\end{array},\right.
\end{gathered}
$$

where $S^{+}(\delta)$ is an LLR value, $S^{\oplus}$ is an element of $\operatorname{GF}(q)$ and $S^{D}[\delta]$ is a binary vector of size $d_{c}$ called Discard Binary Vector (DBV). Fig. 4 shows an example of a $\mathrm{CN}$ for 


\begin{tabular}{|c|c|c|c|}
\hline$U_{1}$ & $U_{2}$ & $U_{3}$ & $U_{4}$ \\
\hline$\left(5, \alpha^{32}\right)$ & $\left(7, \alpha^{12}\right)$ & $\left(4, \alpha^{41}\right)$ & $\left(6, \alpha^{59}\right)$ \\
\hline$\left(4, \alpha^{3}\right)$ & $\left(6, \alpha^{45}\right)$ & $\left(3, \alpha^{33}\right)$ & $\left(5, \alpha^{19}\right)$ \\
\hline$\left(3, \alpha^{21}\right)$ & $\left(5, \alpha^{61}\right)$ & $\left(2, \alpha^{18}\right)$ & $\left(4, \alpha^{46}\right)$ \\
\hline$\left(2, \alpha^{16}\right)$ & $\left(5, \alpha^{41}\right)$ & $\left(1, \alpha^{17}\right)$ & $\left(3, \alpha^{51}\right)$ \\
\hline$\left(0, \alpha^{56}\right)$ & $\left(0, \alpha^{21}\right)$ & $\sqrt{\left(0, \alpha^{21}\right)}$ & $\left(0, \alpha^{15}\right)$ \\
\hline
\end{tabular}

Fig. 4. Example of a deviation path

$q=64, d_{c}=4$ and input messages $U_{i}, i=1, \ldots, d_{c}$ of size $n_{m, i n}=5$. In this figure, the deviation path $\delta=(0,1,0,2)$ is represented by a grey shade in each input vector. It is also represented with straight lines linking $U_{1}[0], U_{2}[1], U_{3}[0]$ and $U_{4}[2]$. Assuming that the elements of $\mathrm{GF}(64)$ are represented by the power of a primitive element $\alpha$, of $\mathrm{GF}(64)$ constructed using the primitive polynomial $P[\alpha]=\alpha^{6}+\alpha+1$, the syndrome associated to $\delta$ is $S(\delta)=\left(0+5+0+4, \alpha^{56} \oplus\right.$ $\left.\alpha^{41} \oplus \alpha^{21} \oplus \alpha^{46}, 0101\right)=\left(9, \alpha^{42}, 0101\right)$.

Let $\Delta_{0}$ be the set of all possible deviation paths that can contribute to an output value, i.e., $\Delta_{0} \subset\left\{0, \ldots, n_{m, i n}-1\right\}^{d_{c}}$. Using the syndrome associated to a deviation path, (3) can be reformulated as

$$
v_{i}^{+}(x)=\min _{\delta \in \Delta_{0}, S^{\oplus}(\delta) \oplus U_{i}^{\oplus}[\delta(i)]=x}\left\{S^{+}(\delta)-U_{i}^{+}[\delta(i)]\right\} .
$$

The DBV is used to reduce the complexity of (9) by avoiding redundant computation. In fact, if $S^{D}[\delta](i)=0$, then $\delta(i)=0$ and $U_{i}^{+}[\delta(i)]=0$. It is thus possible to simplify (9) and express it as

$$
v_{i}^{+}(x)=\min _{\delta \in \Delta_{0}, S^{D}[\delta][i]=0, S^{\oplus}(\delta) \oplus U_{i}^{\oplus}[0]=x}\left\{S^{+}(\delta)\right\} .
$$

Finally, (10) is further reduced by replacing $\delta \in \Delta_{0}$ by $\delta \in \Delta$ where $\Delta$ is a subset of $\Delta_{0}$ with a reduced cardinality $|\Delta|=n_{s}[10]$.

The SB CN algorithm proposed in [10] is summarized in Algo. 1 and its associated architecture is presented in Fig. 5. Step 1 is performed by the Syndrome unit, Step 2 by the Sorting unit and, finally, Step 3 by $d_{c}$ Decorrelation Units (DU) and $d_{c}$ RE units. The DUs are represented in parallel to show the inherent parallelism of the SB CN. The RE units discard couples with a GF value already generated (last test of step 3 in Algo. 1). Note that in [10], the sorting process is done only partially.

Fig. 5 also shows a detailed scheme with the operations in a DU. $S^{D}$ is the $d_{c}$-wide bit vector that indicates for which output edges the syndrome should be discarded during the decorrelation process. A simple reading of bit $i$ in the binary vector $S^{D}$ validates or not the syndrome for the output edge $i$.

\section{Presorting of input messages}

The idea of input presorting is to dynamically change the order of inputs in the $\mathrm{CN}$ processor to classify reliable
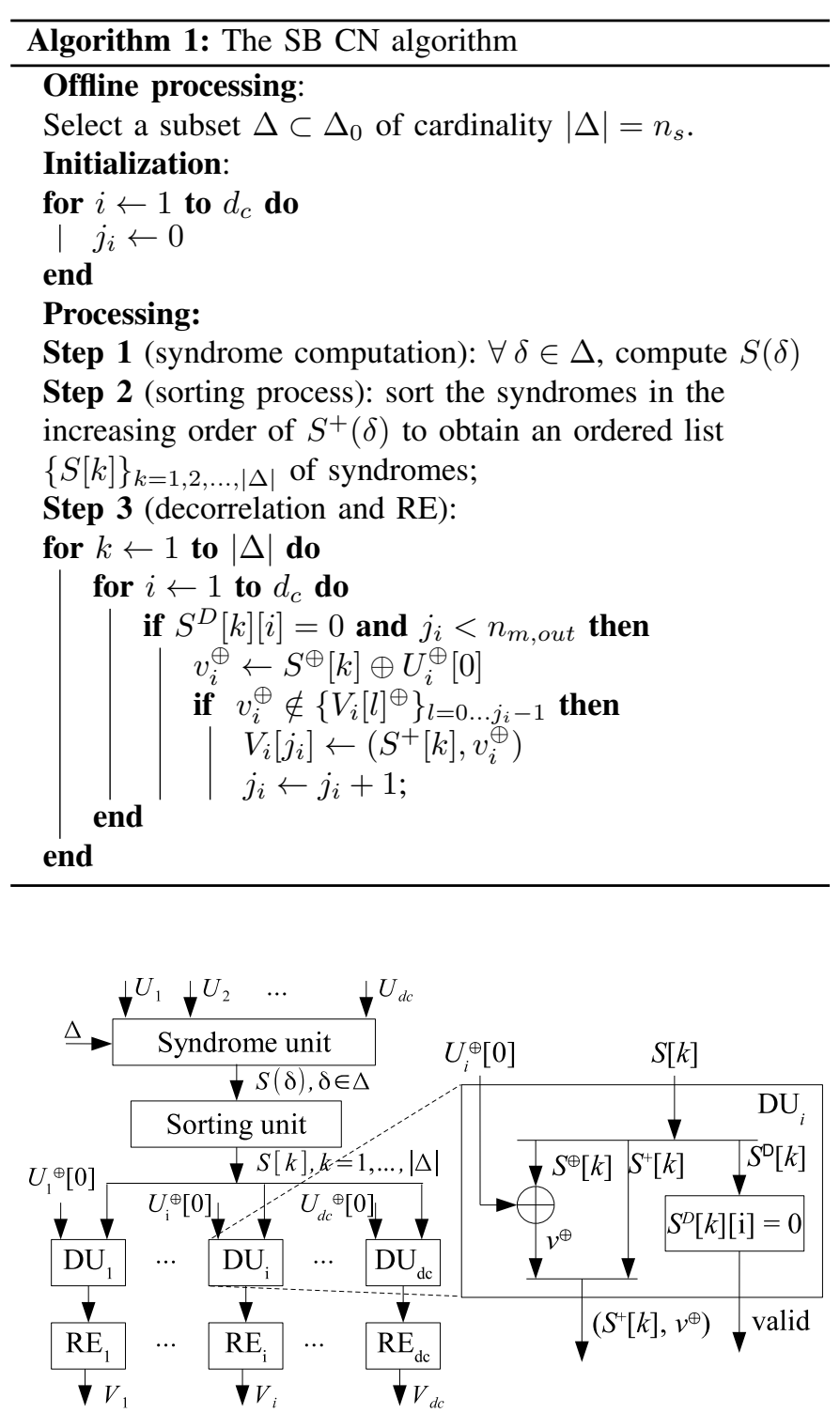

Fig. 5. Syndrome-based CN processing (left part) and details of the DU unit (right part).

and unreliable inputs. This polarization of the inputs makes some deviation paths (for the SB CN [13]) or some potential bubbles (for the FB CN [14]) very unlikely to contribute to an output. The suppression of those useless configurations leads to significant hardware saving without affecting performance.

The presorting principle, as described in Algorithm 2, can be efficiently applied to the EMS algorithm [4] and their derived implementations (FB CN [23][19] and SB CN [10]). Let us consider the application of the presorting algorithm on the EMS-based CN as illustrated in Fig. 6, where $n_{m}=4$ and $d_{c}=4$. In this particular example, the hatched tuples represent tuples that are not involved in the computation of the first 8 syndromes. Compared to the standard $\mathrm{CN}$, the presorting process requires extra hardware: a $d_{c}$-input vector sorter and two permutation networks (or switches). However, it allows some simplifications in the $\mathrm{CN}$ itself, globally leading to an important complexity reduction of the whole $\mathrm{CN}$ processing. 

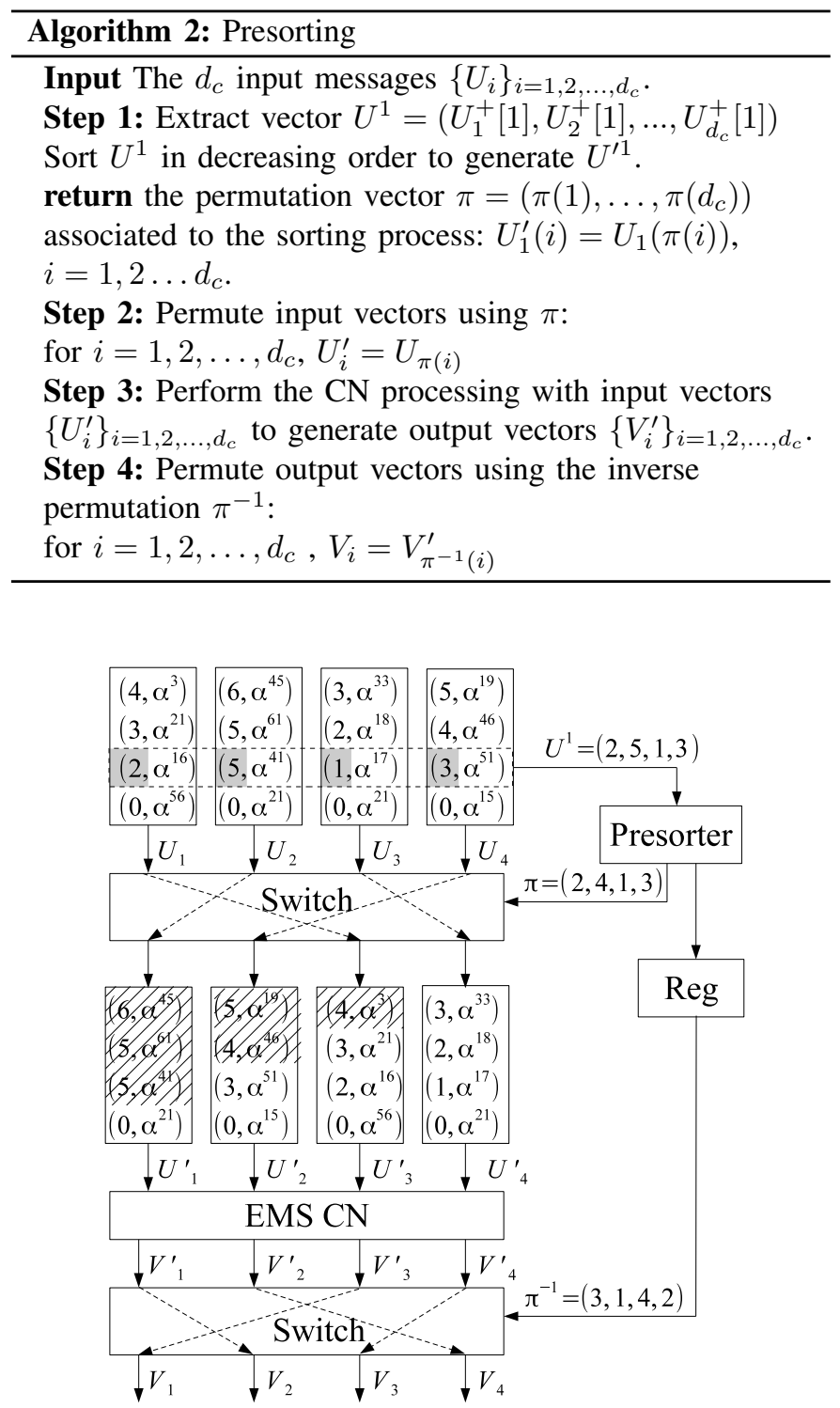

Fig. 6. Presorting principle and EMS-based $\mathrm{CN}$ architecture

\section{Decorrelation and permutation unit}

The first contribution of this paper is to reduce the complexity of the inverse permutation (Step 4 in Algorithm 2) originaly implemented by using a switch as shown at the bottom of Fig. 6. In fact, the decorrelation and switch operations can be efficiently merged for the SB CN architecture. To be specific, the DU can be modified so that it also performs the switching.

This is achieved as follows: note that the Switch block at the bottom of Fig. 6 reorders the $V^{\prime}$ vectors to get the $V$ vectors, i.e. $V(i)=V^{\prime}(\pi(i)), i=1, \ldots, d_{c}$. If the validation test $S^{D}[k][i]=0$ (Fig. 5) in the $i^{\text {th }}$ DU is replaced by $S^{D}[k]\left[\pi^{-1}(i)\right]=0$, then the $i^{t h}$ output will extract the information related to vector $V_{i}$. Note that the GF computation should consider the original input $U_{i}^{\oplus}[0]$ and not $U_{i}^{\prime} \oplus[0]$. The modified DU is named Decorrelation and Permutation Unit (DPU) and an example of its use is shown in Fig. 11.

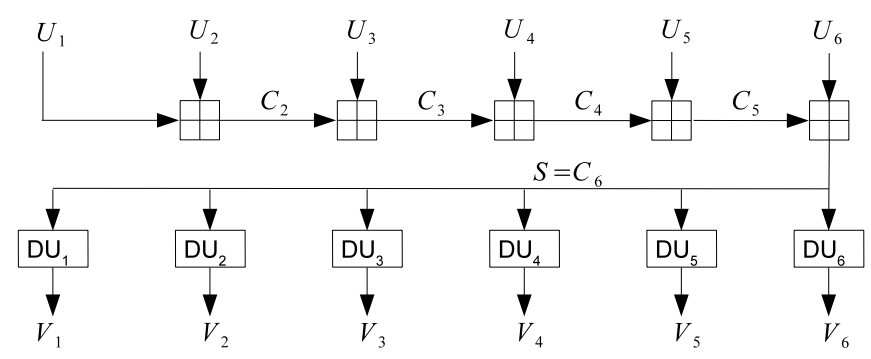

Fig. 7. EF CN Architecture

\section{HYBRID ARCHITECTURES}

In previous Section, the FB and the SB CN architectures as well as the application of the presorting technique to both of them have been presented. In this section, we consider the hybridization of these approaches in a unique $\mathrm{CN}$ architecture. This constitutes the main contribution of this paper. The first proposed hybrid architecture uses an Extended Forward (EF) processing to dynamically generate the set of syndromes. The objective is to take advantage of the simplicity of the SB architecture while keeping the complexity linear with $d_{c}$. The second original architecture introduces presorting in the EF to further reduce the complexity. Finally, a new level of hybridization is performed to take the most advantage of the presorting technique in the $\mathrm{EF}$ architecture.

\section{A. Syndrome computation using the EF processing}

A syndrome set $S_{b}$ can also be computed by performing a forward iteration on all the inputs of the $\mathrm{CN}$ using a serial concatenation of ECNs (5) as

$$
S_{b}=\boxplus_{i=1}^{d_{c}} U_{i} .
$$

Applying the SB CN approach with ECNs of parameters $\left(n_{m}, n_{m}, n_{m}\right)$ provides $n_{m}$ syndromes sorted in increasing order of their associated LLR values. The syndrome set can be computed in a serial scheme as shown in Fig. 7 or it can also be computed with $\left\lceil\log _{2}\left(d_{c}\right)\right\rceil$ layers of ECNs using a tree structure.

Thanks to the use of ECNs, the computed syndrome set is sorted and can be directly applied to the decorrelation process. Note that in [10], a sorting process is required after the syndrome computation. However, the ECNs used in the EF architecture require a small additional patch compared to those in the FB architecture. The role of this patch is to construct the DBV (8) during the ECN processing to provide the additional term $c_{a, b}^{D}$. The ECN addition (4) is then modified as

$$
\begin{aligned}
C_{a, b}=\left(c_{a, b}^{+}, c_{a, b}^{\oplus}, c_{a, b}^{D}\right)= & \left(A^{+}[a]+B^{+}[b],\right. \\
& A^{\oplus}[a] \oplus B^{\oplus}[b], \\
& \left.A^{D}[a] \| B^{D}[b]\right),
\end{aligned}
$$

where $\|$ represents the concatenation operation of two binary vectors.

The CN inputs are initialized with a DBV value of one bit as follows: $U_{i}^{D}[0]=0$ and $U_{i}^{D}[j]=1, \forall j>0$. Thanks to the DBV computation, the output of the EF processing is similar 


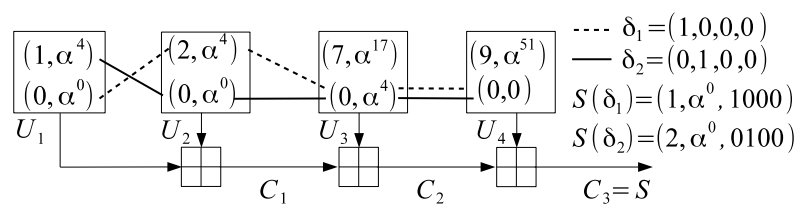

Fig. 8. Example to illustrate the redundant syndromes

to the output of the SB processing just before the decorrelation. In particular, the notion of deviation path can be also applied to the EF processing, with the only difference that the set of deviation paths $\Delta_{E F}$ is input dependent, while $\Delta$ is predefined offline in the SB architecture [10].

A first drawback of the EF is that the number of computed syndromes is typically $3 \times n_{m \text {,out }}$ to compensate the discarded redundant syndromes. Even with this approach, the first simulation results of the EF algorithm showed significant performance degradation compared to the FB algorithm [17]. The reason of this performance degradation resides in the RE process performed by each ECN: since an ECN performs $\mathrm{RE}$, no more than one ECN output could be associated to a given GF value. However, since the ECN outputs in the EF algorithm are partial syndromes, RE may discard useful partial syndromes that would construct valid complete syndromes at the end of the EF processing. In Fig. 8, an example of $\mathrm{CN}$ with $d_{c}=4, n_{m, \text { in }}=2$ and $n_{m, \text { out }}=3$ is presented to illustrate the problem. The two deviation paths $\delta_{1}=(1,0,0,0)$ and $\delta_{2}=(0,1,0,0)$ lead to the same GF value, i.e., $\alpha^{4}+\alpha^{0}+\alpha^{4}+0=\alpha^{0}+\alpha^{4}+\alpha^{4}+0=\alpha^{0}$. The output $C_{1}=U_{1} \boxplus U_{2}$ of the first ECN is equal to $C_{1}=\left\{(0,0,00),\left(1, \alpha^{24}, 10\right),\left(2, \alpha^{24}, 01\right),(3,0,11)\right\}$ before the $\mathrm{RE}$ and equal to $C_{1}=\left\{(0,0,00),\left(1, \alpha^{24}, 10\right)\right\}$ after RE. Note that the seed of the partial syndrome $\delta_{2}$ is eliminated. The final output in this example will be $S=$ $C_{3}=\left\{\left(0, \alpha^{4}, 0000\right),\left(1, \alpha^{0}, 1000\right),\left(7, \alpha^{17}, 0010\right)\right\}$ and after the decorrelation unit, $V_{1}=\left\{\left(0, \alpha^{24}\right),\left(7, \alpha^{47}\right)\right\}$, instead of $V_{1}=\left\{\left(0, \alpha^{24}\right),(2,0),\left(7, \alpha^{47}\right)\right\}$. The key idea to avoid this problem is to allow redundant GF values in the syndrome set. Thus, removing the RE process from the ECN processing avoids performance degradation. Let us define a modified ECN operation with symbol $\boxplus^{\prime}$ where the ECN addition is performed as in (12) and no RE is performed. The syndrome set of size $n_{m}$ can then be computed as

$$
S_{b}^{\prime}=\boxplus_{i=1}^{d_{c}} U_{i}
$$

The RE process will then take place after the decorrelation operation performed by the DUs. As previously mentioned, the set of paths $\Delta$ in the SB CN is pre-determined offline, while it is determined dynamically on the fly in the EF CN according to the current LLR values being processed. This leads to a significant reduction of the total number $n_{s}$ of syndromes to be computed.

\section{B. EF CN with presorting}

As shown in Section III-C, presorting leads to significant hardware savings by reducing the number of candidate GF symbols to be processed within the $\mathrm{CN}$. In this section, we show that this presorting technique, when applied to the message vectors entering the $\mathrm{EF} \mathrm{CN}$, leads to a high complexity reduction of the $\mathrm{CN}$ architecture. This architectural reduction is obtained by reducing the number of bubbles to be considered at each ECN. For this, we perform a statistical study based on Monte-Carlo simulation that traces the paths of the GF symbols that contribute to the output of the $\mathrm{CN}$, in their way across the different ECNs. This statistical study [14] identifies in each ECN how often a given bubble contributes to an output. This information allows pruning the bubbles that never or rarely contribute. More formally, this study is conducted through the following two steps:

1) Monte Carlo simulation giving the trace of the different bubbles (each time a bubble $b$ is used in an output message, its score $\gamma(b)$ is incremented).

2) ECN pruning that aims at discarding the less important bubbles, thus simplifying the ECN architectures.

How to prune low-score bubbles for best efficiency is still an open question. However, we propose here a method that prunes bubbles based on the statistics of their scores at each ECN. Let $I_{b}$ be a sorted set of indexes of the potential bubbles of a given ECN verifying $\forall\left(b, b^{\prime}\right) \in I_{b}^{2}, b<b^{\prime} \Rightarrow \gamma(b) \leq \gamma\left(b^{\prime}\right)$. Let $\tau$ be a real between 0 and 1 and let $\Gamma$ be the cumulative score of all bubbles, i.e., $\Gamma=\sum_{b \in I_{b}} \gamma(b)$. The pruning process suppresses the first $p$ bubbles associated to the first $p$ indexes of $I_{b}$, with $p$ defined as

$$
p=\arg \max _{p^{\prime} \in I_{b}}\left\{\sum_{b=0}^{p^{\prime}} \gamma(b) \leq \tau \Gamma\right\} .
$$

After this pruning process, the structure of some ECNs is greatly simplified. The choice of the values of $\tau$ is a tradeoff between hardware complexity and performance. As an example, Fig. 9 represents the remaining bubbles after the pruning process for a $d_{c}=12 \mathrm{GF}(64)(144,120)$ NB-LDPC code with $n_{m \text {,out }}$ set to 16 . The pruning process has been performed for an SNR of $5 \mathrm{~dB}$ and a value of $\tau$ equal to 0.01 , leading to different simplified ECN architectures:

- 1B: only a single bubble is considered where $C_{i}$ is given by $C_{1}=\left\{\left(0, U_{1}^{\prime \oplus}[0] \oplus U_{2}^{\prime \oplus}[0], 00\right)\right\}$ and $C_{i}=$ $\left\{\left(0, U_{i}^{\prime \oplus}[0] \oplus C_{i-1}^{\oplus}[0], C_{i-1}^{D}[0] \| 0\right)\right\}$ for $i>1$.

- S-1B: it directly generates the $n_{c}^{i}$ sorted output $C_{i}$ as $C_{i}=\left\{\left(0, U_{i}^{\prime \oplus}[0] \oplus C_{i-1}^{\oplus}[0], C_{i-1}^{D} \| 0\right),\left(U_{i}^{\prime+}[b], U_{i}^{\prime \oplus}[b] \oplus\right.\right.$ $\left.\left.C_{i-1}^{\oplus}[0], C_{i-1} \| 1\right)_{b=1 \ldots n_{b}^{i}-1}\right\}$. For this operation, a single GF-adder is required.

- S- $x$ B: with $x>1$, also known as S-bubble ECN. As described in [22], this architecture compares $x$ bubbles per clock cycle.

In Fig. 9, we represent each bubble in an ECN by a filled circle and the direction for the next bubble by an arrow. The size of the arrow is the size of the FIFO in the architecture. Note that the complexity of the ECNs increases from left to right. In fact, only trivial ECN blocks, i.e. $1 \mathrm{~B}, \mathrm{~S}-1 \mathrm{~B}, \mathrm{~S}-2 \mathrm{~B}$ architectures, are required on the left part while a S-5B ECN is required on the right part. It is possible to regroup several ECNs in a single component called Syndrome Node (SN). As detailed hereafter, this $\mathrm{SN}$ computes sorted partial syndromes 


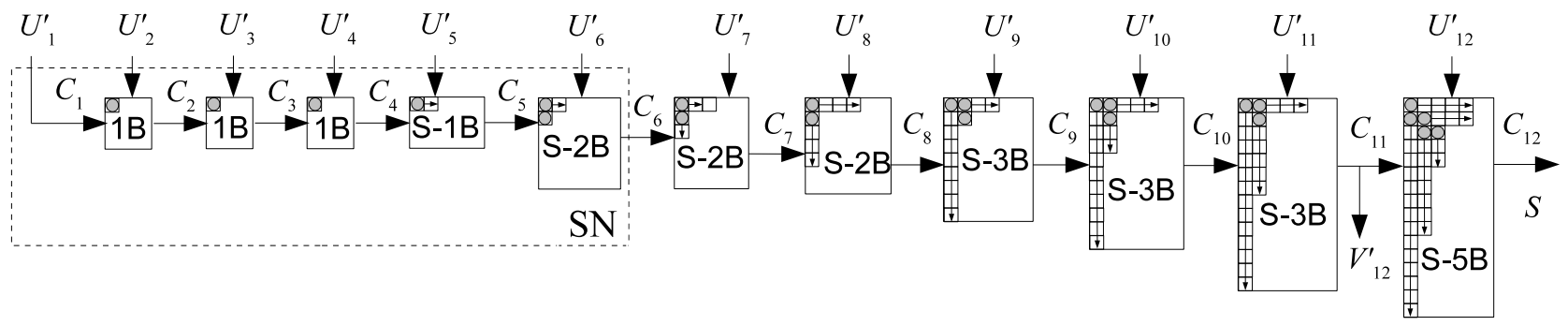

Fig. 9. Architecture of the proposed PS EF CN with $d_{c}=12, n_{b} \leq 4\left(n_{m, i n}=5\right), n_{c}^{12}=n_{s}=20$.

in only one clock cycle, leading to significant hardware and latency reduction.

\section{The Syndrome Node}

In Fig. 9 the first 3 ECNs are of type $1 \mathrm{Bs}$ and can be processed together in a single clock cycle by simply adding the most reliable $\mathrm{GF}$ values of all inputs: $C_{4}=$ $\left\{\left(0, C_{4}^{\oplus}[0], C_{4}^{D}[0]\right)\right\}$, where $C_{4}^{\oplus}[0]=\bigoplus_{i=1}^{4} U_{i}^{\prime \oplus}[0]$ and $C_{4}^{D}[0]=0000$. Also, the $n_{c}^{6}=3$ values of $C_{6}$ can also be computed in one clock cycle. In fact, thanks to presorting, $U_{6}^{\prime+}[1] \leq U_{5}^{\prime+}[1]$ and the first three partial syndromes are

$$
\begin{aligned}
& C_{6}[0]=\left(0, C_{4}^{\oplus}[0] \oplus U_{5}^{\prime \oplus}[0] \oplus U_{6}^{\prime \oplus}[0], 000000\right) \\
& C_{6}[1]=\left(U_{6}^{\prime}[1]^{+}, C_{4}^{\oplus}[0] \oplus U_{5}^{\prime \oplus}[0] \oplus U_{6}^{\prime \oplus}[1], 000001\right) \\
& C_{6}[2]=\left(U_{5}^{\prime}[1]^{+}, C_{4}^{\oplus}[0] \oplus U_{5}^{\prime \oplus}[1] \oplus U_{6}^{\prime \oplus}[0], 000010\right),
\end{aligned}
$$

In summary, we can consider all these computations to belong to a unique block, i.e. the $\mathrm{SN}$, that involves several ECNs (to be specific, 5 ECNs in the example of Fig. 9) but that generates its outputs in a single clock cycle.

\section{Hybridization between $F B$ and EF $C N$ architectures}

Combining the EF architecture and the FB approach leads to a reduction of the total number of needed syndromes to guarantee a given number of valid syndromes. Fig. 10 shows the average number of syndromes that should be computed for a given output $V_{i}^{\prime}$ to obtain, with a probability of $90 \%$, $n_{o p}=18$ valid syndromes . This number is denoted by $n_{s}^{0.9}(i)$ and varies for each output $V_{i}^{\prime}$. Note that when the presorting technique is considered, $n_{s}^{0.9}(i)$ increases with $i$. To decode without performance degradation, the number of computed syndromes is bounded by the number of syndromes required by the last output, i.e. $n_{s}=n_{s}^{0.9}(12)=46$ in the example of Fig. 10.

Fig. 9 shows that $V_{12}^{\prime}$ can be directly obtained from $C_{11}$ without DU, as $C_{11}$ contains the contribution of all the inputs except $U_{12}^{\prime}$, i.e. $V_{12}^{\prime}=C_{11}$. This result can be seen as the application of the FB algorithm on the output $V_{12}^{\prime}$ since the forward process of the FB algorithm is included in the EF systematically genetating $V_{12}^{\prime}$. Consequently, the number of required syndromes can be reduced from $n_{s}(12)=46$ to $n_{s}(11)=36$, since $V_{12}^{\prime}$ is computed directly. This reduces the overall complexity and latency of the $\mathrm{EF} \mathrm{CN}$ architecture

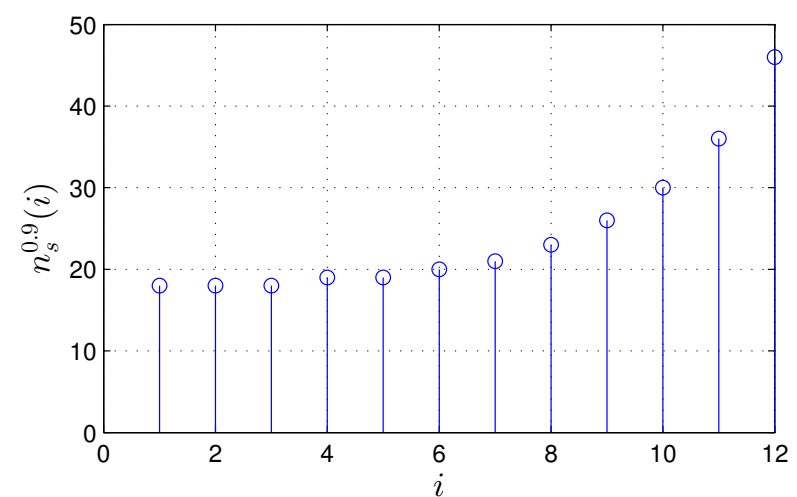

Fig. 10. Maximum number of syndromes needed to be generate, for each output $V_{i}^{\prime}, n_{o p}=18$ valid syndromes. The output number is denoted by $i$. The code rate is $R=5 / 6$ and $\mathrm{SNR}=4.5 \mathrm{~dB}$.

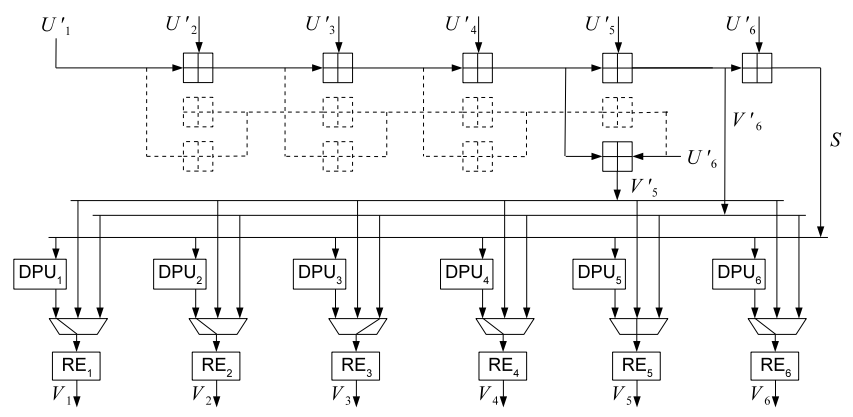

Fig. 11. $\operatorname{HB}(0,4,2)$ architecture for a $\mathrm{CN}$ with $d_{c}=6$. The last two outputs $V_{5}^{\prime}$ and $V_{6}^{\prime}$ are generated by a classical FB architecture.

without performance degradation. Note that this constitutes a first example of a hybrid architecture where one output is generated with the FB approach and the other $d_{c}-1=11$ outputs with the EF CN. This kind of approach can be generalized, as described by the following.

\section{E. General notations for hybrid architectures}

Let $\operatorname{HB}\left(\rho_{S N}, \rho_{E F}, \rho_{F B}\right)$ be an hybrid architecture that combines the SN, EF and FB schemes. The first $\rho_{S N}$ inputs are processed by a SN block, the next $\rho_{E F}$ inputs are processed by an EF block and the remaining $n_{F B}$ inputs are processed by a FB block. Obviously, $\rho_{S N}+\rho_{E F}+\rho_{F B}=d_{c}$.

Fig. 11 shows the $\operatorname{HB}(0,4,2)$ architecture for a $\mathrm{CN}$ of degree 6. As shown, $V_{5}^{\prime}$ and $V_{6}^{\prime}$ are computed using the FB algorithm in order to further reduce the number of required 
syndromes. There are several possible $\mathrm{HB}$ architectures between the $\mathrm{EF}$ (i.e., $\operatorname{HB}(0,6,0)$ ) and the classical $\mathrm{FB} \mathrm{CN}$ (i.e., $\operatorname{HB}(0,0,6))$. Note that $V_{6}^{\prime}$ (resp. $\left.V_{5}^{\prime}\right)$ should bypass the decorrelation units and should be directly connected to $V_{\pi(6)}$ (resp. $\left.V_{\pi(5)}\right)$. Fig. 11 shows the case where $\pi(6)=3$, i.e. the third multiplexer connects $V_{3}$ to $V_{6}^{\prime}$, and $\pi(5)=5$, i.e. the fifth multiplexer connects $V_{5}$ to $V_{6}^{\prime}$. Finally, $V_{1}, V_{2}, V_{4}$ and $V_{6}$ are each one connected to the output of the corresponding DPU. Fig. 13 shows the $\operatorname{HB}(6,4,2)$ architecture for a $\mathrm{CN}$ of degree 12. $V_{11}^{\prime}$ and $V_{12}^{\prime}$ are computed using the $\mathrm{FB}$ algorithm and a $\mathrm{SN}$ is used to process the 6 first input $U_{1}^{\prime}$ to $U_{6}^{\prime}$.

\section{F. Choice of parameters $\left(\rho_{S N}, \rho_{E F}, \rho_{F B}\right)$}

The determination of the $\mathrm{CN}$ architecture parameters, i.e. $\left(\rho_{S N}, \rho_{E F}, \rho_{F B}\right)$ for the macro level, the internal structure of the EF and the FB blocks (the parameters of each ECN) for the micro level, is a complex problem. It can be formulated as an optimization problem: how to minimize the hardware complexity without introducing significant performance degradation. In this paper, we have limited the value of $\rho_{F B}$ to 1 and 2 . Then, for the two hypotheses $\left(0, d_{c}-\rho_{F B}, \rho_{F B}\right)_{\rho_{F B}=1,2}$, we have applied the same method than the one described in Section IV-B to determine the parameters of each ECN of the EF and FB blocks. Note that after the automatic raw pruning process described in Section IV-B, the parameters are further tuned by hand by a "try and see (i.e. estimate performance by simulation)" method. Once the pruning process finished, the value of $\rho_{S N}$ is fixed in order to optimize the hardware efficiency of the $\mathrm{CN}$ architecture. In fact, at a given point, $\mathrm{CN}$ with parameters $\left(\rho_{S N}+1, \rho_{E F}-1, \rho_{F B}\right)$ will have a higher hardware complexity than $\mathrm{CN}$ with parameters $\left(\rho_{S N}, \rho_{E F}, \rho_{F B}\right)$ but with a lower decoding latency.

\section{G. Suppression of final output RE}

In some decoder implementations [16] [20] with $d_{v}=2$, the VNs connected to a $\mathrm{CN}$ are updated just after the $\mathrm{CN}$ update. For example, in Fig. 11 a variable node unit may be connected directly to each output $V_{1}$ to $V_{6}$ just after the $\mathrm{RE}$ units $\mathrm{RE}_{1}$ to $\mathrm{RE}_{6}$. Also in some $\mathrm{VN}$ implementations [16] [20], RE is performed in the VN. If this type of VN is used, then the RE block can be removed from the hybrid architecture for complexity reduction. The suppression of the $d_{c}$ RE block is interesting specially for for high $d_{c}$ values. In a $\mathrm{HB}$ architecture with final RE, the RE reduces the number of output messages from $n_{s}$ (in case that all messages are valid) to $n_{m \text {,out }}$. Although removing the RE results in an increase of the number of output messages $\left(n_{s}>n_{m, \text { out }}\right)$, it has a limited impact on the complexity since the $n_{m \text {,out }}$ elements are processed on the fly by the VN, without the need of intermediate storage. However, it may impact slightly the $\mathrm{VN}$ power consumption since $n_{s}$ elements are processed instead of $n_{\text {m,out }}$. Note that the suppression of RE neither affects the algorithm nor the performance since the RE is still performed in the VN.

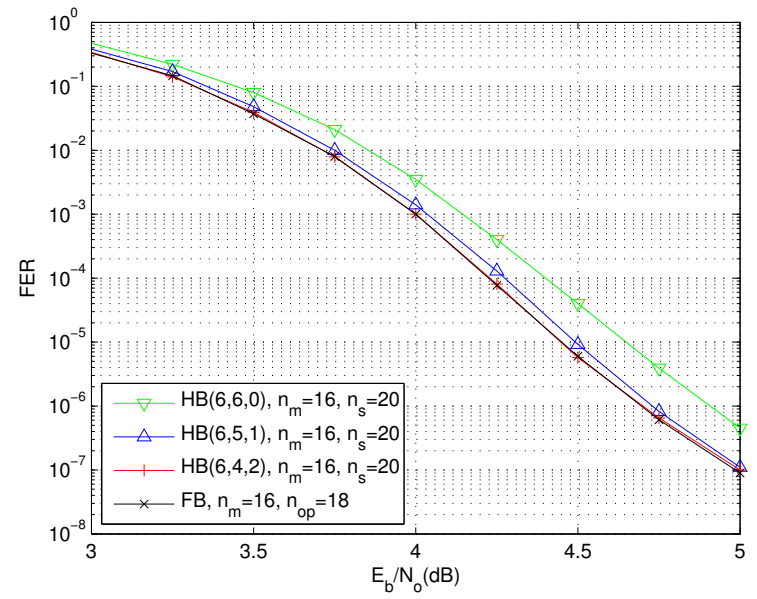

Fig. 12. FER performance for a $(144,120)$ NB-LDPC code over GF(64).

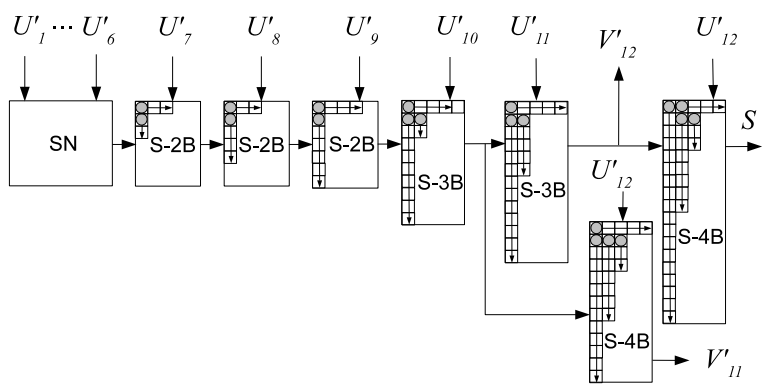

Fig. 13. $\operatorname{HB}(6,4,2)$ architecture with $d_{c}=12, n_{m, \text { out }}=16, n_{m, \text { in }}=5$ and $n_{s}=20$.

\section{PERFORMANCE AND COMPLEXITY ANALYSIS}

We consider GF(64)- and GF(256)-LDPC codes to obtain performance and post-synthesis results for the different proposed decoding architectures.

\section{A. Performance}

We ran bit-true Monte-Carlo simulations over the Additive White Gaussian Noise channel (AWGN) with a Binary Phase Shift Keying (BPSK) modulation scheme. The different parameters were set as follows: extrinsic and intrinsic LLR messages quantified on 6 bits, the a posteriori LLRs on 7 bits and the maximum number of decoding iterations to 10 . The matrices used in our simulations are available in [24].

Fig. 12 shows the obtained Frame Error Rate (FER) for a GF(64) code of size $(864,720)$ bits, code rate $R=5 / 6$, $d_{c}=12$ and $d_{v}=2$ over the Gaussian channel. We consider the FB decoder in [22] as a reference, i.e. S-bubble algorithm with 4 bubbles, $n_{m}=16$ and $n_{o p}=18$. We simulated the $\operatorname{HB}(6,6,0)$ or $\mathrm{EF}$, the $\operatorname{HB}(6,5,1)$ and the $\operatorname{HB}(6,4,2)$ architectures with the same number of computed syndromes $n_{s}=20$. Fig. 13 shows the $\operatorname{HB}(6,4,2)$ architecture, for which no performance degradation is observed. We observe less than $0.05 \mathrm{~dB}$ of performance loss for the $\operatorname{HB}(6,5,1)$ and around $0.2 \mathrm{~dB}$ for the $\operatorname{HB}(6,6,0)$ configuration. We then conclude from these simulation results that the hybrid architectures can achieve the same performance as the FB 


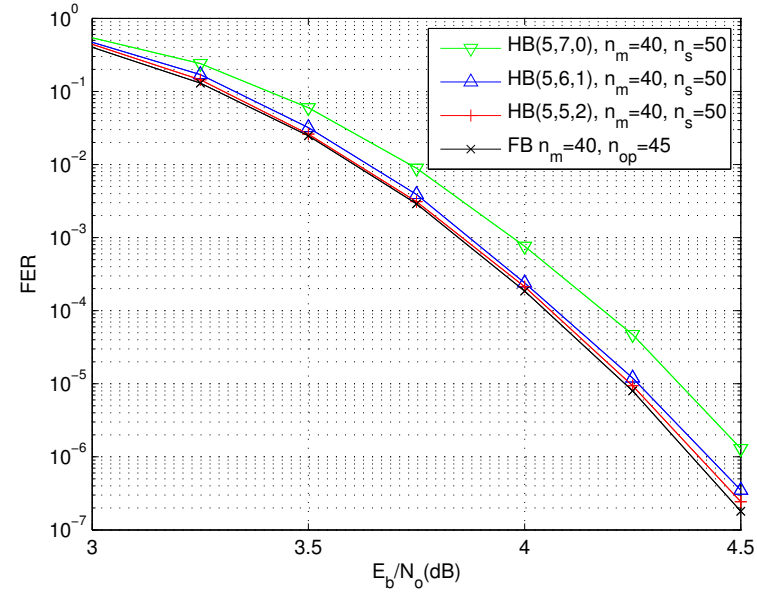

Fig. 14. FER performance for a $(144,120)$ NB-LDPC code over GF(256).

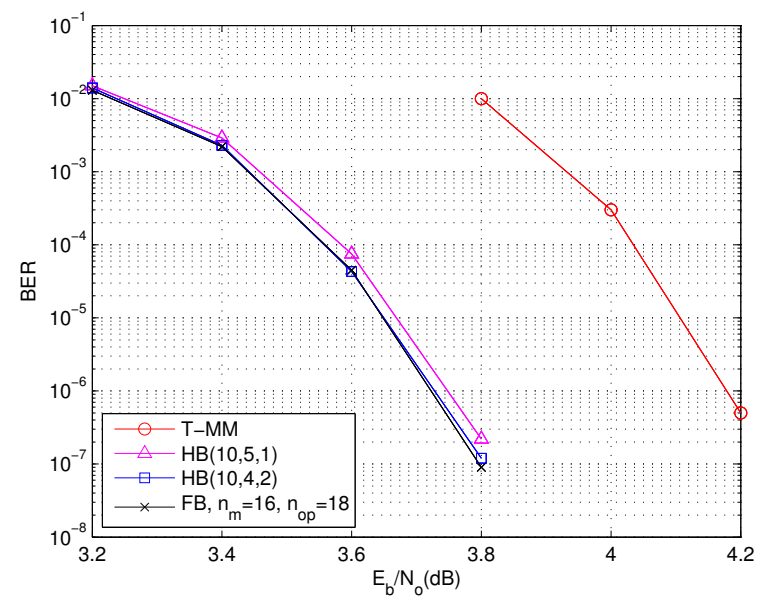

Fig. 15. BER performance for a $(1536,1344)$ NB-LDPC code over GF(64).

architecture and outperform the EF architecture while needing a reduced number of syndromes with a factor ranging from 3 to 4 .

Fig. 14 shows performance results for a GF(256)-LDPC code of size $(1152,960)$ bits, code rate $R=5 / 6, d_{c}=12$ and $d_{v}=2$. We consider as a reference the FB decoder with a S-bubble architecture [22], 6 bubbles, $n_{m}=40$ and $n_{o p}=45$. The $\operatorname{HB}(5,5,2)$ architecture presents the same performance as the $\mathrm{FB}$ and the $\operatorname{HB}(5,6,1)$ shows slight performance loss smaller than $0.05 \mathrm{~dB}$. The EF architecture presents around 0.1 $\mathrm{dB}$ of performance loss compared to the FB.

We can then conclude that this new family of hybrid architectures allows for significant complexity reduction in $\mathrm{CN}$ implementations without any performance loss compared to more complex state-of-the-art solutions.

Fig. 15 shows performance results for a $\mathrm{GF}(64)$ (1536, 1344) LDPC code, code rate $R=7 / 8, d_{c}=16$ and $d_{v}=2$. As reference, we consider the FB decoder with a S-bubble architecture [22], 4 bubbles, $n_{m}=16$ and $n_{o p}=18$. The architecture being used is the same as the one presented in Fig. 13 except for the fact that the SN includes four more
TABLE II

POST-SYNTHESIS RESULTS FOR DIFFERENT ECN ARCHITECTURES AND CN SUB-UNITS ON 28 NM FD-SOI TECHNOLOGIE.

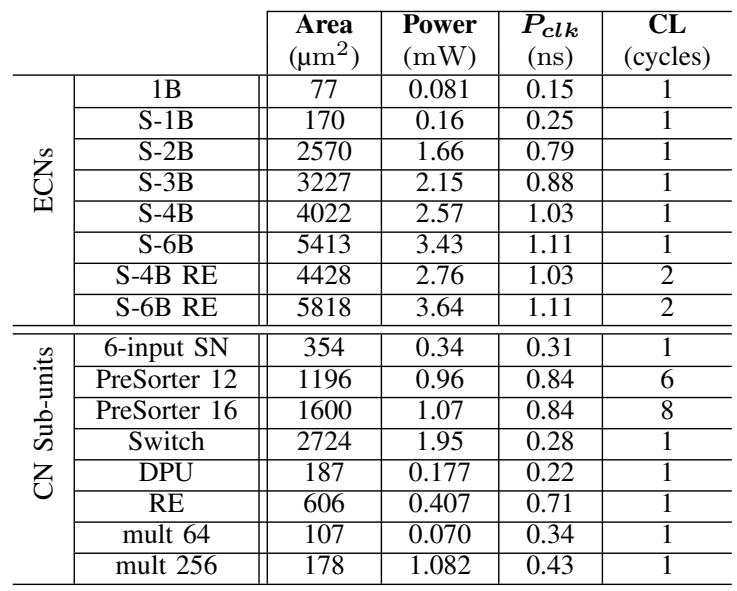

1B ECNs (i.e., the $\mathrm{HB}(10,4,2) \mathrm{CN}$ architecture). The TMM performance of a code of same length and coding rate provided in [8] is also presented in Fig. 15. The Hybrid architecture shows a performance gain of $0.5 \mathrm{~dB}$ over the $\mathrm{T}$ MM architecture. However, the performance gap may come from the use of different parity check matrices. In [8] the authors consider a $d_{c}=24, d_{v}=3$ code with an array dispersion construction while we use a $d_{c}=16, d_{v}=2$ code constructed using optimized coefficients for the parity checks [25].

\section{B. Implementation results}

For complexity and power analysis, we considered the implementation of the architectures on $28 \mathrm{~nm}$ FD-SOI technologies targeting a clock frequency of $800 \mathrm{MHz}$. The different kinds of ECNs presented in Fig. 9, were synthesized individually to provide the results in Table II. Additionally, the synthesis results of the $\mathrm{SN}$, the S-bubble with RE (used in the FB CN), the sorter, the switch, the DU (Fig. 5) and the RE units implementation are also provided. The sorter is implemented using a serial architecture as in [14], and the switch is a cross bar switch. The minimum clock period $\left(P_{c l k}\right)$ is given in nanoseconds with a clock uncertainty of $0.05 \mathrm{~ns}$ and a setup time of $0.02 \mathrm{~ns}$. The Cycle Latency (CL) represents the number of clock cycles between the first input and the first output. A reduction factor of 57,34 and 7 is observed between the $1 \mathrm{~B}$ and the S-4B RE architectures in terms of area, power and clock period, respectively. These results show that significant gain can be obtained thanks to ECN simplification even if it implies the overcost of the presorter, the switch and the DPU units.

Table III summarizes the implementation results for all the $\mathrm{CN}$ architectures presented in this paper, for a GF(64) and a GF(256)-LDPC codes with $d_{c}=12$. In this Table we present the synthesis results with and without RE considering that the RE can be suppressed in implementations when $d_{v}=2$ [16] [20]. CL(CN) gives the latency in cycles between the first input of a $\mathrm{CN}$ and the first output of a $\mathrm{CN}$, taking into account the latency of the ECNs, the Pre-Sorter, the switch, the DPU, the 
TABLE III

POST-SYNTHESIS RESULTS FOR CN ARCHITECTURES

\begin{tabular}{|c|c|c|c|c|c|}
\hline GF & CN & $\begin{array}{c}\text { Area } \\
\left(\mathrm{mm}^{2}\right)\end{array}$ & $\begin{array}{l}\text { Power } \\
(\mathrm{mW})\end{array}$ & $\begin{array}{c}P_{c l k} \\
(\mathrm{~ns})\end{array}$ & $\begin{array}{c}\mathbf{C L}(\mathbf{C N}) \\
\text { (cycles) }\end{array}$ \\
\hline \multirow{15}{*}{64} & $\overline{\mathrm{FB}}$ & $\overline{0.140}$ & $\overline{944}$ & 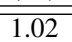 & $\overline{22}$ \\
\hline & PS FB & 0.037 & 26 & 1.12 & 20 \\
\hline & EF RE & 0.125 & 83 & 1.22 & 13 \\
\hline & PS EF & 0.0328 & 26 & 1.12 & 19 \\
\hline & PS EF RE & 0.037 & 28 & 1.12 & 19 \\
\hline & $\mathrm{HB}(6,6,0)$ & 0.0227 & 14.9 & 1.03 & 15 \\
\hline & $\mathrm{HB}(6,6,0) \mathrm{RE}$ & 0.0269 & 17.1 & 1.03 & 15 \\
\hline & $\mathrm{HB}(0,10,2)$ & 0.0257 & 16.5 & 1.17 & 19 \\
\hline & $\mathrm{HB}(0,10,2) \mathrm{RE}$ & 0.0292 & 18.4 & 1.17 & 19 \\
\hline & $\mathrm{HB}(6,5,1)$ & 0.0228 & 15.2 & 1.04 & 15 \\
\hline & $\mathrm{HB}(6,5,1) \mathrm{RE}$ & 0.0271 & 17.4 & 0.99 & 15 \\
\hline & $\mathrm{HB}(6,4,2)$ & 0.0259 & 19 & 1.00 & 15 \\
\hline & $\mathrm{HB}(6,4,2) \mathrm{RE}$ & 0.0306 & 19.4 & 0.99 & 15 \\
\hline & $\mathrm{HB}(10,4,2)$ & 0.0307 & 30 & 0.99 & 17 \\
\hline & $\mathrm{HB}(10,4,2) \mathrm{RE}$ & 0.0363 & 35 & 0.95 & 17 \\
\hline \multirow{7}{*}{256} & $\overline{\mathrm{FB}}$ & 0.328 & $\overline{210}$ & 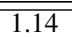 & 22 \\
\hline & PS EF & 0.074 & 54 & 1.06 & 19 \\
\hline & PS EF RE & 0.0909 & 65 & 1.17 & 19 \\
\hline & $\mathrm{HB}(5,6,1)$ & 0.0753 & 45 & 1.2 & 16 \\
\hline & $\mathrm{HB}(5,6,1) \mathrm{RE}$ & 0.0871 & 48 & 1.15 & 16 \\
\hline & $\mathrm{HB}(5,5,2)$ & 0.0803 & 45.4 & 1.20 & 16 \\
\hline & $\mathrm{HB}(5,5,2) \mathrm{RE}$ & 0.094 & 52 & 1.20 & 16 \\
\hline
\end{tabular}

$\mathrm{RE}$ and the GF multiplication and division. For the FB architecture, $\mathrm{CL}(\mathrm{CN})=\mathrm{CL}($ mult $)+\left(d_{c}-2\right) \times \mathrm{CL}(\mathrm{S}-4 \mathrm{~B}) \mathrm{RE}+$ $\mathrm{CL}($ div $)=22$. For the $\operatorname{HB}(6,4,2)$ architecture (Fig. 13), $\mathrm{CL}(\mathrm{CN})=\mathrm{CL}($ Sorter $)+\mathrm{CL}($ Switch $)+\mathrm{CL}(\mathrm{SN})+3 \times$ $\mathrm{CL}(\mathrm{S}-2 \mathrm{~B})+2 \times \mathrm{CL}(\mathrm{S}-3 \mathrm{~B})+\mathrm{CL}(\mathrm{S}-4 \mathrm{~B})+\mathrm{CL}(\mathrm{DPU})=$ 14 , considering that the multiplication is performed in the same cycle as the switch and the division is performed in the same cycle as the DPU.

For GF(256) results, the FB architecture is with $n_{m}=40$ and S-6B ECNs, the EF and HB architectures consider ECNs with a maximum $n_{m, i n}$ value of 6 .

\section{Area and energy efficiency comparison}

To compare the efficiency of the different $\mathrm{CN}$ architectures, we consider the number of computed $\mathrm{CNs}$ per second as follows: $T_{C N}=1 /\left(\mathrm{CL}\right.$ (layer) $\left.\times P_{c l k}\right)$ where $\mathrm{CL}$ (layer) is the periodicity of a $\mathrm{CN}$ computation in a layered decoder. In our design, $\mathrm{CL}($ layer $)=\mathrm{CL}(\mathrm{CN})+\mathrm{CL}(\mathrm{VN})+n_{m, \text { out }}+n_{m, \text { int }}$ where $\mathrm{CL}(\mathrm{VN})$ is $7, n_{m, i n t}=4$ for $\mathrm{GF}(64)$ and $n_{m, i n t}=6$ for $\mathrm{GF}(256)$. The Area Efficiency (AE) can then be expressed as: $\mathrm{AE}=T_{C N} /$ Area in computed $\mathrm{CN}$ per second per $\mathrm{mm}^{2}$. The Energy Efficiency (EE) can be expressed as EE = $F_{c l k} /($ Power $\times \mathrm{CL}($ layer $)) \mathrm{CN}$ computation per micro joule where the clock frequency $F_{c l k}$ is set at $800 \mathrm{MHz}$. In Table IV, we compare the different architectures. The $\operatorname{HB}(6,5,1)$ and $\mathrm{HB}(6,4,2)$ in $\mathrm{GF}(64)$ improve the $\mathrm{AE}$ compared with the $\mathrm{FB}$ architecture by a factor of 6.8 and 6.2, respectively. When comparing the EE, the improvement factors are of 6.4 and 5.5.

The CN implementation results of Table III and Table IV compare the $\mathrm{FB}, \mathrm{EF}$ and $\mathrm{HB}$ architectures with and without RE. To compare the HB to the SB, we refer to [26]. Table V presents the obtained post-synthesis results. The complexity is expressed in terms of NAND gates in order to make a fair comparison between the two different ASIC technologies. For
TABLE IV

AREA AND ENERGIE EFFICIENCY FOR DIFFERENT ARCHITECTURES

\begin{tabular}{c|c||c|c|c|c}
\hline \multirow{2}{*}{ GF } & $\mathbf{C N}$ & $\begin{array}{c}\text { CL(layer) } \\
\text { (cycles) }\end{array}$ & $\begin{array}{c}\boldsymbol{T}_{\boldsymbol{C N}} \\
\left(\frac{\mathrm{Mcn}}{\mathrm{s}}\right)\end{array}$ & $\begin{array}{c}\mathbf{A E} \\
\left(\frac{\mathrm{Mcn}}{\mathrm{mm}^{2} \mathrm{~s}}\right)\end{array}$ & $\begin{array}{c}\mathbf{E E} \\
\left(\frac{\mathrm{Mcn}}{\mathrm{mJ}}\right)\end{array}$ \\
\hline \hline \multirow{4}{*}{64} & $\mathrm{FB}$ & 47 & 20.8 & 148 & 0.18 \\
\cline { 2 - 6 } & $\mathrm{PS}$ FB & 45 & 19.8 & 535 & 0.68 \\
\cline { 2 - 6 } & $\mathrm{PS}$ EF & 46 & 19.0 & 579 & 1.45 \\
\cline { 2 - 6 } & $\mathrm{HB}(6,5,1)$ & 42 & 23.1 & 1013 & 1.25 \\
\cline { 2 - 6 } & $\mathrm{HB}(6,4,2)$ & 42 & 23.8 & 919 & 1.00 \\
\cline { 2 - 6 } & $\mathrm{HB}(10,4,2)$ & 44 & 22.7 & 739 & 0.61 \\
\hline \hline \multirow{3}{*}{256} & $\mathrm{FB}$ & 74 & 11.8 & 36 & 0.051 \\
\cline { 2 - 6 } & $\mathrm{HB}(5,6,1)$ & 69 & 12.1 & 160 & 0.257 \\
\cline { 2 - 6 } & $\mathrm{HB}(5,5,2)$ & 69 & 12.1 & 151 & 0.255 \\
\hline
\end{tabular}

TABLE V

HB AND SB COMPARISON

\begin{tabular}{c||c|c|c|c|c|c}
\hline CN & $\begin{array}{c}\text { Tech. } \\
(\mathrm{nm})\end{array}$ & $\boldsymbol{d}_{\boldsymbol{c}}$ & $\begin{array}{c}\boldsymbol{F}_{\text {clk }} \\
(\mathrm{MHz})\end{array}$ & $\begin{array}{c}\text { Gates } \\
(\mathrm{M} \text { NAND) }\end{array}$ & $\begin{array}{c}\boldsymbol{T}_{\boldsymbol{C N}} \\
\left(\frac{\mathrm{Mcn}}{\mathrm{s}}\right)\end{array}$ & $\begin{array}{c}\mathbf{A E} \\
\left(\frac{\mathrm{Mcn}}{\text { MNAND }}\right)\end{array}$ \\
\hline \hline SB & 65 & 4 & 400 & 0.058 & 100 & 1724 \\
\hline SB & 65 & 12 & 280 & 0.13 & 18 & 138 \\
\hline \hline HB & 28 & 12 & 800 & 0.053 & $9.5^{*}$ & $180^{*}$ \\
\hline HB & 28 & 16 & 800 & 0.063 & $9.1^{*}$ & $144^{*}$ \\
\hline * A clock frequency of $F_{c l k}=400 \mathrm{MHz}$ is considered.
\end{tabular}

$T_{C N}$ and $\mathrm{AE}$ computation, a clock frequency of $F_{c l k}=400$ $\mathrm{MHz}$ is considered to compare the different technologies.

Focusing on $\mathrm{AE}$ as a function of $d_{c}$ for $\mathrm{HB}$ and $\mathrm{SB}$, one can note that $\mathrm{AE}$ of $\mathrm{HB}$ slightly decrease with $d_{c}$ while $\mathrm{AE}$ of SB decreases significantly with $d_{c}$. The $d_{c}$ threshold for which HB becomes more efficient than SB can be estimated at $d_{c}=12$ from a linear interpolation of obtained result.

\section{Comparison with the T-MM architecture}

For a fair comparison with the T-MM architecture [8] [9], the full decoder is considered since the T-MM-base decoder uses a different $\mathrm{VN}$ architecture. We implemented a complete decoder architecture based on an $\mathrm{HB}(10,4,2) \mathrm{CN}$ over $\mathrm{GF}(64)$ which is connected to $16 \mathrm{VNs}$. The variable node units mainly consist in adding $n_{m, \text { out }}$ values with their corresponding intrinsics and then sorting the $n_{m, i n}$ smallest obtained values. A decision unit is implemented in parallel to each $\mathrm{VN}$ in order to implement the stopping criteria. The synthesis results are given in Table VI. We considered the same clock frequency as [8] $F_{c l k}=350 \mathrm{MHz}$ to compute the $\mathrm{HB}$ efficiency and throughput for a fair comparison. Those results triggers us few comments, first, this comparison is not really fair for TMM since a higher variable and check node degree is used for T-MM $\left(d_{v}=3, d_{c}=24\right)$ compared with HB architecture $\left(d_{v}=2, d_{c}=16\right)$. Second, the variable nodes of the Hybrid architecture are not yet fully optimized and further potential gains are expected. A more general comparison between the two architectures for several Galois Field orders ranging from $\mathrm{GF}(64)$ to $\mathrm{GF}(1024)$ would be very interesting.

\section{E. Throughput}

The throughput can be greatly improved by processing $p$ CNs in parallel in a layered decoder [27] [16]. Because we consider a code with $d_{v}=2$, the VNs are cascaded after the $\mathrm{CN}$ as in [16] [20]. The throughput of a layered decoder is 
TABLE VI

POST-SYNTHESIS RESULTS OF HB $(10,4,2)$ DECODER FOR $(1512,1323)$ NB-LDPC CODE OVER GF(64)

\begin{tabular}{c||c|c|c}
\hline & T-MM [9] & T-MM [8] & HB $(10,4,2)$ \\
\hline \hline Technologie $(\mathrm{nm})$ & 90 & 90 & 28 \\
\hline$F_{c l k}(\mathrm{MHz})$ & 270 & 350 & 800 \\
\hline Gate count $(\mathrm{M}$ NAND) & 2.09 & 2.99 & 1.0 \\
\hline Thoughput (Mbps) & 1403 & 1231 & $267^{*}$ \\
\hline Efficiency $(\mathrm{Mbps} / \mathrm{M}$ gates) & 671 & 412 & $222^{*}$ \\
\hline * clock frequency of $F_{c l k}=350 \mathrm{MHz}$ is considered.
\end{tabular}

given by $T=\left(N_{b} \times F_{c l k} \times p\right) /\left(M \times \mathrm{CL}(\right.$ layer $\left.) \times i t_{a v r}\right)$ where $i t_{a v r}$ is the average number of iterations and $F_{c l k}$ is fixed at $800 \mathrm{MHz}$. The layered $\mathrm{GF}(64)(1536,1344)$ code allows different parallelism options depending on the splitting factor [28] ( $p=2,3,4,6,8,12,16,24,32,48,96)$. With an observed average number of iterations at SNR $=4.0$ of 1.5 and a parallelism of 12 , the throughput can reach $1.2 \mathrm{Gbps}$ with $p=2$ and 7.3 Gbps with $p=12$.

\section{CONCLUSION}

This paper was dedicated to low-complexity implementations of $\mathrm{CN}$ processors in NB-LDPC decoders. We reviewed the state-of-the-art architectures that consider the Extended Min-Sum algorithm and introduced new approaches to reduce the hardware complexity of the $\mathrm{CN}$ processors. We particularly focused on the effect of the presorting technique and the advantages of the Extended-Forward architecture. We then presented the hybrid architectures which combine the ExtendedForward and the Forward-Backward approaches to significantly reduce the total number of computed syndromes. The post-synthesis results on $28 \mathrm{~nm}$ ASIC technology showed that the area efficiency is improved by a factor of 6.2 without any performance loss, or by a factor of 6.8 with a performance loss of $0.04 \mathrm{~dB}$ compared with the Forward-Backward architecture.

\section{REFERENCES}

[1] R. Gallager, "Low-density parity-check codes," Ph.D. dissertation, Cambridge, 1963.

[2] M. C. Davey and D. J. C. MacKay, "Low density parity check codes over GF(q)," in Information Theory Workshop, 1998, Jun 1998, pp. 70-71.

[3] S. Pfletschinger and D. Declercq, "Getting closer to MIMO capacity with non-binary codes and spatial multiplexing," in Global Telecommunications Conference (GLOBECOM 2010), 2010 IEEE, Dec 2010, pp. $1-5$.

[4] D. Declercq and M. Fossorier, "Decoding algorithms for nonbinary LDPC codes over GF(q)," IEEE Transactions on Communications, vol. 55, no. 4, pp. 633-643, April 2007.

[5] A. Voicila, D. Declercq, F. Verdier, M. Fossorier, and P. Urard, "Lowcomplexity decoding for non-binary LDPC codes in high order fields," IEEE Transactions on Communications, vol. 58, no. 5, pp. 1365-1375, May 2010.

[6] E. Li, D. Declercq, and K. Gunnam, "Trellis-based extended Min-Sum algorithm for non-binary LDPC codes and its hardware structure," IEEE Transactions on Communications, vol. 61, no. 7, pp. 2600-2611, July 2013.

[7] J. O. Lacruz, F. García-Herrero, J. Valls, and D. Declercq, "One minimum only trellis decoder for non-binary low-density parity-check codes," IEEE Transactions on Circuits and Systems I: Regular Papers, vol. 62 , no. 1, pp. 177-184, Jan 2015

[8] J. O. Lacruz, F. García-Herrero, M. J. Canet, and J. Valls, "Reducedcomplexity nonbinary LDPC decoder for high-order Galois fields based on trellis Min-Max algorithm," IEEE Transactions on Very Large Scale Integration (VLSI) Systems, vol. 24, no. 8, pp. 2643-2653, Aug 2016.
[9] H. P. Thi and H. Lee, "Basic-set trellis Min-Max decoder architecture for nonbinary LDPC codes with high-order Galois fields," IEEE Transactions on Very Large Scale Integration (VLSI) Systems, vol. 26, no. 3, pp. 496-507, March 2018.

[10] P. Schläfer, N. Wehn, M. Alles, T. Lehnigk-Emden, and E. Boutillon, "Syndrome based check node processing of high order NB-LDPC decoders," in Telecommunications (ICT), 2015 22nd International Conference on, April 2015, pp. 156-162.

[11] P. Schläfer, V. Rybalkin, N. Wehn, M. Alles, T. Lehnigk-Emden, and E. Boutillon, "A new architecture for high throughput, low latency NB-LDPC check node processing," in Personal, Indoor, and Mobile Radio Communications (PIMRC), 2015 IEEE 26th Annual International Symposium on, Aug 2015, pp. 1392-1397.

[12] V. Rybalkin, P. Schläfer, and N. Wehn, "A new architecture for high speed, low latency NB-LDPC check node processing for GF(256)," in 2016 IEEE 83rd Vehicular Technology Conference (VTC Spring), May 2016, pp. 1-5.

[13] C. Marchand and E. Boutillon, "NB-LDPC check node with pre-sorted input," in 9th International Symposium on Turbo Codes and Iterative Information Processing (ISTC), Sept 2016, pp. 196-200.

[14] H. Harb, C. Marchand, A. A. Ghouwayel, L. Conde-Canencia, and E. Boutillon, "Pre-sorted forward-backward NB-LDPC check node architecture," in IEEE International Workshop on Signal Processing Systems (SiPS), Oct 2016, pp. 142-147.

[15] Y. L. Ueng, K. H. Liao, H. C. Chou, and C. J. Yang, "A high-throughput trellis-based layered decoding architecture for non-binary LDPC codes using max-log-QSPA," IEEE Transactions on Signal Processing, vol. 61, no. 11, pp. 2940-2951, June 2013.

[16] C. L. Lin, S. W. Tu, C. L. Chen, H. C. Chang, and C. Y. Lee, "An efficient decoder architecture for nonbinary LDPC codes with extended min-sum algorithm," IEEE Transactions on Circuits and Systems II: Express Briefs, vol. 63, no. 9, pp. 863-867, Sept 2016.

[17] C. Marchand, H. Harb, E. Boutillon, A. Al Ghouwayel, and L. CondeCanencia, "Extended-forward architecture for simplified check node processing in NB-LDPC decoders," in IEEE International Workshop on Signal Processing Systems (SiPS), October 2017.

[18] C. Poulliat, M. Fossorier, and D. Declercq, "Design of regular $(2, \mathrm{dc})-$ LDPC codes over GF(q) using their binary images," IEEE Transactions on Communications, vol. 56, no. 10, pp. 1626-1635, October 2008.

[19] A. Voicila, D. Declercq, F. Verdier, M. Fossorier, and P. Urard, "Lowcomplexity decoding for non-binary LDPC codes in high order fields," IEEE Transactions on Communications, vol. 58, no. 5, pp. 1365-1375, May 2010.

[20] E. Boutillon, L. Conde-Canencia, and A. A. Ghouwayel, "Design of a GF(64)-LDPC decoder based on the EMS algorithm," IEEE Transactions on Circuits and Systems I: Regular Papers, vol. 60, no. 10, pp. 2644-2656, Oct 2013

[21] Y. S. Park, Y. Tao, and Z. Zhang, "A fully parallel nonbinary LDPC decoder with fine-grained dynamic clock gating," IEEE Journal of SolidState Circuits, vol. 50, no. 2, pp. 464-475, Feb 2015.

[22] O. Abassi, L. Conde-Canencia, A. A. Ghouwayel, and E. Boutillon, "A novel architecture for elementary-check-node processing in nonbinary LDPC decoders," IEEE Transactions on Circuits and Systems II: Express Briefs, vol. 64, no. 2, pp. 136-140, Feb 2017.

[23] H. Wymeersch, H. Steendam, and M. Moeneclaey, "Log-domain decoding of LDPC codes over GF(q)," in Communications, 2004 IEEE International Conference on, vol. 2, June 2004, pp. 772-776 Vol.2.

[24] UBS. (2018) Non Binary LDPC. [Online]. Available: http: //www-labsticc.univ-ubs.fr/nb_ldpc/

[25] E.Boutillon, "Optimization of non binary parity check coefficients," IEEE Transactions on Information Theory, 2018, submitted. [Online]. Available: https://arxiv.org/abs/1708.01761

[26] P. Schläfer, "Implementation aspects of binary and non-binary lowdensity parity-check decoders," Ph.D. dissertation, Technische Universitt Kaiserslautern, 2016.

[27] Y. L. Ueng, C. Y. Leong, C. J. Yang, C. C. Cheng, K. H. Liao, and S. W. Chen, "An efficient layered decoding architecture for nonbinary QCLDPC codes," IEEE Transactions on Circuits and Systems I: Regular Papers, vol. 59, no. 2, pp. 385-398, Feb 2012.

[28] C. Marchand, J. B. Dore, L. Conde-Canencia, and E. Boutillon, "Conflict resolution for pipelined layered LDPC decoders," in 2009 IEEE Workshop on Signal Processing Systems, Oct 2009, pp. 220-225. 


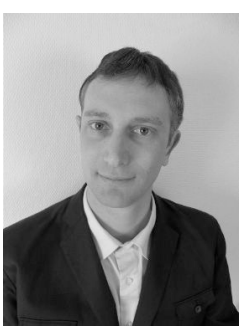

Cédric Marchand was born in 1976, France. He received his B.E. degree in electrical and electronics engineering from the North East Wales Institute, Wrexhan, Wales, 1999, M.Sc. and Ph.D. degrees in electrical engineering from the Université Européenne de Bretagne in 2007 and 2011 respectively. From 2007 to 2011, he has been working with NXP Semiconductor France on the implementation of an LDPC decoder for the DVB-S2, -T2 and -C2 standards. In 2012, he joined Université Bretagne Sud as research engineer. His current research interests include decoding architectures for error correcting codes and VLSI design.

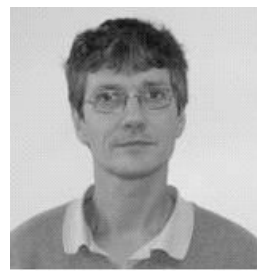

Emmanuel Boutillon was born in Chatou, France in 1966. He received the Engineering Diploma from Telecom Paris Tech, Paris, France in 1990. In 1991, he worked as an assistant professor in the Ecole Multinationale Supérieure des Télécommunications in Africa (Dakar). In 1992, he joined Telecom Paris Tech as a research engineer where he conducted research in the field of VLSI for digital communications. He obtained his Ph.D in 1995. In 1998, he spent a sabbatical year at the University of Toronto, Ontario, Canada. In 2000, he joined Université Bretagne Sud as a professor. He headed the LESTER lab from 2005 up to end of 2007 and the CACS department between 2008 up to 2015 . He is currently the scientific advisor of the Lab-STICC. In 2011, he had a sabbatical year at INICTEL-UNI, Lima (Peru). His research interests are on the interactions between algorithm and architecture in the field of wireless communications and high speed signal processing. In particular, he works on Turbo Codes and LDPC decoders.

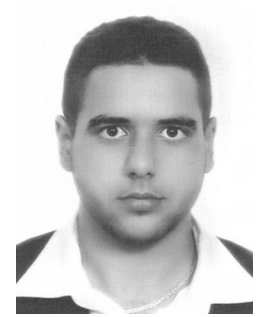

Hassan Harb was born in 1992, Lebanon. He received the Master 2 degree in electronics and telecommunications from the Lebanese University, Lebanon, Beirut, 2015. Currently he is a PhD student in cooperation between Université Bretagne Sud, Lorient, France and Lebanese University, Beirut, Lebanon. He is working on the correction code called NB-LDPC algorithm, design and implementation.

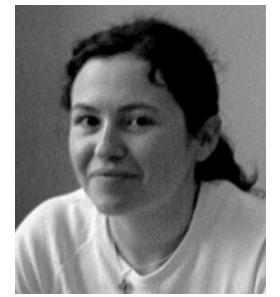

Laura Conde-Canencia received Eng. Degree from Universidad Politenica de Madrid, Spain, in 2000 and Ph.D. from Telecom Bretagne, France, in 2004. Since 2006 she is with the Lab-STICC Laboratory in Université Bretagne Sud (UBS), France, where she is currently an Associate Professor. She is a member of the Scientific Advisory Board of UBS and a member of the International Relations Advisory Board. In 2006-2007, she worked on PN sequence synchronization for Global Navigation Satellite System receivers. Since 2008, her research has mainly focused on error correcting codes for digital communications: Turbo-codes, binary and non-binary LDPC codes and Cortex codes. From 2013 she has extended her research topics to neural coding and neuromorphic design. Since 2016 she is an elected member of the "Design and Implementation of Signal Processing Systems" Technical Committee (IEEE Signal Processing society). In 2017-2018, she was a Fulbright Visiting Research Scholar at University of California Los Angeles (UCLA). During this year she was co-funded by the European Union with a PRESTIGE grant award or Marie Curie Fellowship.

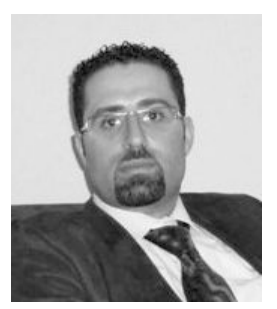

Ali Al Ghouwayel received his B.E. degree in Physics-Electronics in 2002 from the Lebanese University, Beirut, his B.E. degree in Electronics Engineering and his M.S. Degree in Telecommunications in 2004 from the National School of Engineering of Brest (ENIB), Brest, France, and his $\mathrm{PhD}$ in Signal Processing and Telecommunications in 2008 from the High School of Electricity (Suplec) and University of Rennes 1, Rennes, France. His research activities concerned Parametrization Study for Software Radio Systems. In 2008, He joined the Lab-STICC laboratory in Lorient, France and worked as a Post-Doctoral researcher on the European Project DAVINCI. In 2010, He joined the Lebanese International University, in Beirut, Lebanon, as Assistant Professor in the Computer and Communication Engineering Department. His current research interests include Study, Optimization, and Adequation AlgorithmArchitecture for Hardware Implementation of Non-Binary LDPC decoders, and Design of Reconfigurable Ultra-Throughput Rate Architectures for 5G Systems. 